\title{
Clinical evaluation of the rapid recovery of patients who underwent video-assisted thoracoscopic lung surgery under non-intubated anesthesia
}

\author{
Lin-Sheng Cai ${ }^{1 \#}$, Bo Hou ${ }^{2 \#}$, Hua Jin ${ }^{3}$, Yun Bo ${ }^{3}$, Xin-Long Chen ${ }^{4}$, Jun Dai ${ }^{4}$, Tao Yang ${ }^{4}$, Bao-Shi Lan ${ }^{5}$, \\ Jia Ye ${ }^{1}$, Hao Peng ${ }^{4}$, Jun Peng ${ }^{4}$
}

${ }^{1}$ Medical School of Kunming University of Science and Technology, Kunming, China; ${ }^{2}$ Department of Thoracic Surgery, The First People's Hospital of Qujing, Qujing, China; ${ }^{3}$ Department of Anesthesiology, The First People's Hospital of Yunnan Province, The Affiliated Hospital of Kunming University of Science and Technology, Kunming, China; ${ }^{4}$ Department of Thoracic Surgery, The First People's Hospital of Yunnan Province, The Affiliated Hospital of Kunming University of Science and Technology, Kunming, China; ${ }^{5}$ Department of Thoracic Surgery, The People's Hospital of Panzhou City, Panzhou, China

Contributions: (I) Conception and design: LS Cai, B Hou, H Peng, J Peng; (II) Administrative support: LS Cai, B Hou, H Jin, Y Bo, H Peng, J Peng; (III) Provision of study materials or patients: LS Cai, B Hou, XL Chen, H Jin, H Peng, J Peng; (IV) Collection and assembly of data: LS Cai, J Dai, T Yang, BS Lan, J Ye; (V) Data analysis and interpretation: LS Cai, H Jin, H Peng, J Peng; (VI) Manuscript writing: All authors; (VII) Final approval of manuscript: All authors.

\#These authors contributed equally to this work.

Correspondence to: Jun Peng; Hao Peng. Department of Thoracic Surgery, The First People's Hospital of Yunnan Province, the Affiliated Hospital of Kunming University of Science and Technology, Kunming, China. Email: phao9375@163.com; 389647518@qq.com.

Background: Non-intubated anesthesia thoracoscopic surgery is an evolving form of minimally invasive thoracic surgery. It has had encouraging results in the treatment of lung cancer, and the current concept of enhanced recovery after surgery has become indispensable to surgical treatment. Our center retrospectively evaluated the clinical effect of rapid postoperative rehabilitation in patients who underwent thoracoscopic lung surgery under non-intubated anesthesia.

Methods: The clinical data of 192 patients undergoing video-assisted thoracoscopic surgery (VATS) at the Department of Thoracic Surgery of The First People's Hospital of Yunnan Province were retrospectively analyzed. Among them, 75 received double-lumen endotracheal intubation anesthesia, and 117 received a laryngeal mask instead of endotracheal intubated anesthesia. Comparing the intra-and post-operative indicators difference of these 2 groups.

Results: There were no significant differences in the postoperative complications, length of hospital stay, hemoglobin counts and platelet count $24 \mathrm{~h}$ after surgery between the non-intubated anesthesia and intubated anesthesia groups $(\mathrm{P}>0.05)$. In terms of the postoperative rehabilitation indicators of the VATS patients, the non-intubated anesthesia group had an earlier recovery in terms of water (food) intake, out-ofbed activity, and defecation (exhaust) times $(\mathrm{P}<0.001)$, less postoperative complications and adverse throat reactions $(\mathrm{P}<0.05)$, and higher levels of satisfaction $(\mathrm{P}<0.05)$ than the intubated anesthesia group. Compared to the intubated anesthesia group, the non-intubated anesthesia group had fewer changes of white blood cell counts, neutrophil percentages and lymphocytes percentages $24 \mathrm{~h}$ postoperatively $\left(9.25 \times 10^{9}\right.$ vs. $7.67 \times 10^{9}$, $\mathrm{P}=0.045 ; 7.06$ vs. 5.53, $\mathrm{P}=0.039 ; 1.45$ vs. $1.27, \mathrm{P}=0.017)$, significant reduction pleural drainage after restoring oral intake in postoperative 24 and $48 \mathrm{~h}$ (198.43 vs. 131.07 and 114.04 vs. $57.47 \mathrm{~mL}, \mathrm{P}<0.001)$, shorter postoperative thoracic tube drainage times (3.73 vs. $3.13 \mathrm{~d}, \mathrm{P}<0.001)$. Notably, there was no difference between the smoking history and previous medical history of the patients in the 2 groups.

Conclusions: Compared to intubation by general anesthesia, non-intubated thoracoscopic anesthesia is a safe and effective option. Patients who undergo non-intubated thoracoscopic lung surgery have a rapid recovery after surgery. Thus, it is worthy of promotion in clinical surgery settings. 
Keywords: Video-assisted thoracoscopic surgery (VATS); non-intubated anesthesia; enhanced recovery after
surgery (ERAS); inflammatory response

Submitted Oct 29, 2021. Accepted for publication Dec 14, 2021.

doi: $10.21037 / \mathrm{atm}-21-6434$

View this article at: https://dx.doi.org/10.21037/atm-21-6434

\section{Introduction}

Video assisted thoracic surgery (VATS) gradually be popularized in the past 3 decades, the way of minimally invasive surgery has become a thoracic surgery treatment of lung cancer is the most commonly choice (1). The traditional general anesthesia VATS achieved pulmonary surgery by endotracheal intubation with one-side lung ventilation, endotracheal intubation caused many adverse reactions for rehabilitation of postoperative patients as invasive device $(2,3)$. Non-intubated VATS became the focus of many scholars. Non-intubated VATS, i.e., thoracoscopic surgery without endotracheal intubation by general anesthesia with spontaneous ventilation, is a new approach to this technique of minimally invasive surgery $(4,5)$. Encouragingly, non-intubated anesthesia VATS optimized anesthesia and surgery, enhance recovery after surgery and early hospital discharge, reduce hospitalization cost, etc. $(6,7)$, its clinical outcomes are also significant part of the rapid recovery strategy (8).

In recent years, with the popularization of the concept of enhanced recovery after surgery (ERAS), ERAS strategy, i.e., is a concept of perioperative management, promoting early discharge from hospital for reduce morbidity and costs of the patients ERAS can minimize complications and accelerate recovery by preoperative optimization and evidence-based clinical measures etc. $(9,10)$. Non-intubated VATS have been achieved many clinical rehabilitation advantages, the clinical application of non-intubated anesthesia VATS is still controversial and has only been popularized in some research centers. The combination of non-intubated anesthesia VATS lung surgery with ERAS accelerates postoperative recovery less have reported, and its clinical feasibility still deserved to discuss. We performed non-intubated anesthesia VATS lung surgery, and better initial effects were obtained (11). We analyzed the clinical feasibility of rapid postoperative recovery in 192 patients underwent non-intubated anesthesia VATS lung surgery in our center. We present the following article in accordance with the STROBE reporting checklist (available at https:// dx.doi.org/10.21037/atm-21-6434).

\section{Methods}

\section{Study subjects}

\section{Inclusion indicators}

The clinical data of thoracic surgery patients from September 2014 to December 2020 were retrospectively collected. One hundred ninety-two patients were identified who underwent thoracoscopic lung surgery. The patients were divided into an intubated anesthesia group (the control group) and a non-intubated anesthesia group (and experimental group). All procedures performed in this study involving human participants were in accordance with the Declaration of Helsinki (as revised in 2013). Ethical approval for the study was granted by the Ethics Committee of The First People's Hospital of Yunnan Province (KHLL2021-KY091). In addition, written informed consent was obtained from all patients who participated in the study. To be eligible for inclusion in the study, patients had to meet the following inclusion criteria: baseline data (e.g., gender, age, past history and smoking history of patients).

Clinical indicators were observed that lists the intraoperative blood loss $(\mathrm{mL})$, postoperative evaluation indexes \{e.g., time to water intake/food intake [b], outof-bed activity time [ $b]$, returning time of exhaust/ defecation $[d]$, laryngeal pain or discomfort, nausea or vomiting, postoperative complications, satisfaction degree), inflammatory indexes $24 \mathrm{~h}$ after surgery (routine blood), chest drainage changes 24 and $48 \mathrm{~h}$ after oral recovery, and the number of drainage days $[d]$.

\section{Exclusion indicators}

Patients were excluded from the study if they met any of the following exclusion criteria: (I) had a contraindication to an anesthesia; (II) had a body mass index (BMI) $>30 \mathrm{~kg} / \mathrm{m}^{2}$; (III) had a difficult airway; (IV) had preoperative severe cardiopulmonary complications; (V) rejected the anesthesia plan, or had a surgical contraindication; and/or (VI) hypoxemia $\left(\mathrm{PaO}_{2}<60 \mathrm{mmHg}\right)$ or hypercapnia $\left(\mathrm{PaCO}_{2}\right.$ $>50 \mathrm{mmHg}$ ) preoperatively; (VII) had underwent a previous pulmonary resection (12), see Table 1. 
Table 1 Exclusion criteria for non-intubated thoracoscopic surgery

Exclusion criteria
$\mathrm{BMI}>30 \mathrm{~kg} / \mathrm{m}^{2}$
Difficult airway
Severe cardiopulmonary dysfunction
Patient rejection of anesthesia plan
Persistent cough or chronic cough with large amount of airway
secretion
Hypoxemia $\left(\mathrm{PaO}{ }_{2}<60\right.$ mmHg) or hypercapnia
$\left(\mathrm{PaCO} \mathrm{mm}_{2}>50 \mathrm{~mm}\right)$ preoperatively
Underwent a previous pulmonary resection

BMI, body mass index.

\section{Anesthesia and surgical methods}

Non-intubation anesthesia groups: after the intravenous injection of etomidate, midazolam, sufentanil, and atropine, a laryngeal mask airway was inserted by manual-assisted ventilation or synchronous intermittent mechanical ventilation (SIMV). The parameters of the anesthetic ventilator (oxygen flow rate $3-5 \mathrm{~mL} / \mathrm{min}$ and $\mathrm{SpO}_{2}>90 \%$ ) were evaluated and adjusted. Propofol and a remifentanil intravenous pump was used during the operation to maintain deep anesthesia, which was discontinued immediately after the operation. The chest wall incision was selected pleural cavity at the 4th or 5th intercostal space of the midaxillary line of the affected side that cut into pleural cavity, and promote the affected side lung tissue spontaneously collapse by cooperating of the anesthesiologist. The thoracic cavity was explored by dissecting and separating the surrounding tissues of focus. An endoscopic cutting and closing device were used to assist with the excision. The lung tissue was dissected, or the hilar lung and the mediastinal lymph nodes were dissected according to the intraoperative freezing pathology results. At the end of the operation, normal saline was injected into the thoracic cavity, and a lung expansion test was performed with the assistance of the anesthesiologist to confirm that there was no air leakage at the broken end of the bronchus. The thoracic drainage tube was left in the operation hole, and the chest was closed. After the patient recovered from the anesthesia, the patient was returned to the ward and the postoperative indicators were monitored.

Intubation anesthesia groups: anesthetic drugs, midazolam, etomidate, atropine and vecuronium bromide were injected into the peripheral vein. Double-lumen endotracheal intubation was performed after manualassisted ventilation or SIMV. The intraoperative anesthesia management and operation methods were the same as those used in the non-intubation group (see above). Patients in both groups recovered well after surgery, and there were no cases of death or reoperation.

\section{Statistical methods}

SPSS23.0 software was used for the statistical analysis. The continuous data are expressed as means \pm standard deviations. The $\chi^{2}$ test was used to examine the categorical data. The Mann-Whitney U-test was used to examine differences between the 2 groups. P value $<0.05$ was considered statistically significant.

\section{Results}

\section{Patient characteristics and postoperative adverse events}

The basic clinical data of 192 patients with pulmonary diseases who underwent VATS surgery between September 2014 and December 2020 were compared. Seventy-five patients received intubation under general anesthesia, and 117 patients received non-intubated anesthesia. There were no differences between the smoking histories and postoperative complications of the 2 groups. The degree of pharyngeal pain (or discomfort) and the degree of nausea or vomiting were lower in the non-intubated group than the intubated group. The differences in basic clinical data between the 2 groups are shown in Table 2 .

\section{Analysis of intra- and post-operative indicators}

The amount of intraoperative blood loss and the length of hospital stay were compared between the 2 groups (15.37 vs. $16.63 \mathrm{~d}, \mathrm{P}=0.091 ; 86.87$ vs. $84.8 \mathrm{~mL}, \mathrm{P}<0.001)$. The postoperative recovery indicators were compared between the 2 groups, including the resumption of the oral intake of water and out-of-bed activity (3.52 vs. 1.12, $11.54 v s .2 .23$, $\mathrm{P}<0.001)$. The postoperative satisfaction of non-intubated group compared to which intubated group was better and the exhaust time was shortened (96.18 vs. $99.8 \%, \mathrm{P}<0.001$; 1.11 vs. $1.01, \mathrm{P}=0.004)$. In the 2 groups, the total thoracic drainage volume reduced more significantly in the nonintubation group than in the intubation group (667.24 vs. $440.65 \mathrm{~mL}, \mathrm{P}=0.005)$, the postoperative thoracic tube drainage time was shorter ( 3.73 vs. $3.13 \mathrm{~d}, \mathrm{P}<0.001$; see Table 3). 
Table 2 The clinical data of the 2 groups, and the statistical test results comparing the groups

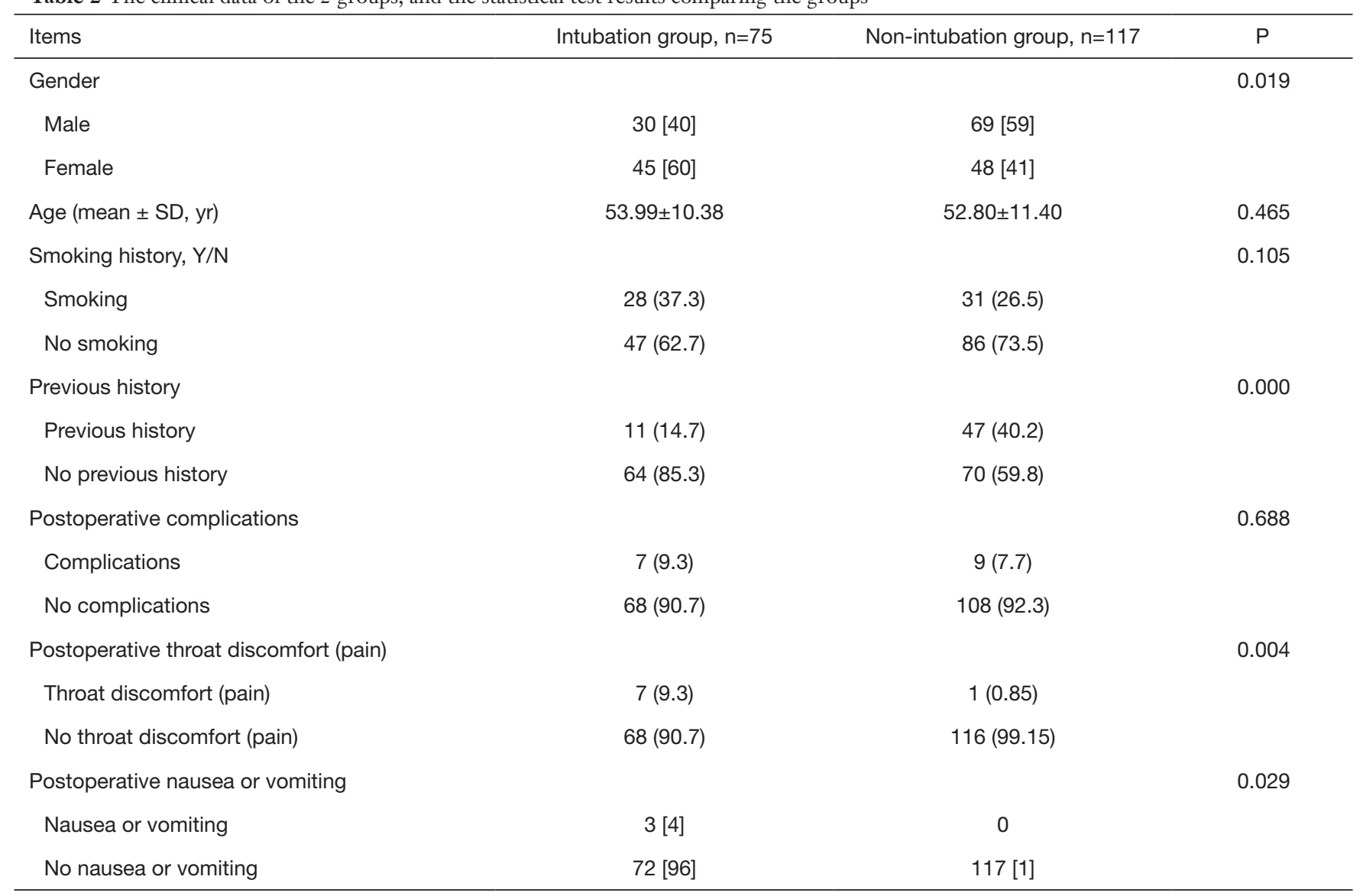

Continuous data are shown as the mean \pm standard deviation, and categorical variables as the number (\%).

\section{Postoperative blood routine and pleural drainage indexes}

Compared with intubation group, leukocyte (WBC), neutrophil (NEUT), and lymphocyte (LYMPH), hemoglobin (HB) counts decreased non-intubated group $24 \mathrm{~h}$ after surgery $\left(9.25 \times 10^{9}\right.$ vs. $7.67 \times 10^{9}, \mathrm{P}=0.045 ; 7.06$ vs. $5.53, \mathrm{P}=0.039 ; 1.45$ vs. $1.27, \mathrm{P}=0.017)$. The changes inhemoglobin (HB) and platele (PLT) were not significant (126.93 vs. $120.45, \mathrm{P}=0.213 ; 229.65$ vs. $216.51, \mathrm{P}=0.212$ ). Notably, the pleural drainage fluid of non-intubated group progressively decreased 24 and $48 \mathrm{~h}$ after restoring oral intake (198.43 vs. $131.07 \mathrm{~mL}, 114.04$ vs. $57.47 \mathrm{~mL}, \mathrm{P}<0.001$; see Table 4).

\section{Discussion}

At present, non-intubated anesthesia VATS is a new option for minimally invasive surgery of thoracic surgery. Lung surgery under spontaneous ventilation is closer to physiological characteristics, the reduction of anesthetic drugs and requires surgeons to achieve surgery more accurately and rapidly, optimizing anesthesia and surgical techniques, less invasive injuries, faster recovery, reduced postoperative complications, accelerated the rapid recovery and early discharge of postoperative patients (13). Comparing with intubation anesthesia VATS lung surgery, limitations of non-intubated anesthesia VTAS; including: (I) cough reflex, (II) mediastinal movement under spontaneous breathing, (III) persistent hypoxemia and hypercapnia during the operation, (IV) anesthesiologists and surgeons need tacit cooperation, etc. (14). These problems became the focus of discussion. We found that non-intubated anesthesia VATS lung surgery reduced postoperative complications and throat adverse reaction. Laryngeal mask ventilation and vagus nerve block advantageous to overcome these risk factors, our center achieved a 92-year-old patient who underwent non-intubation anesthesia VAST lung 
Table 3 Intraoperative and postoperative clinical indexes $(\bar{x} \pm \mathrm{s})$

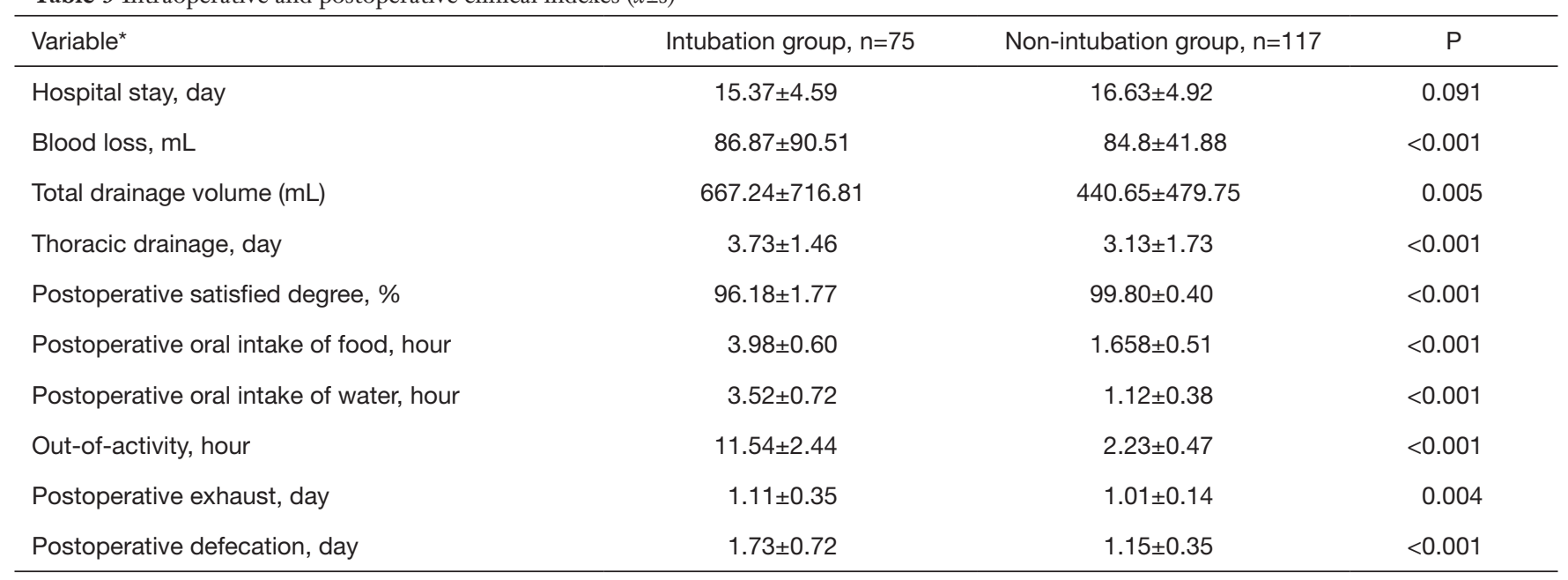

${ }^{*}$, the continuous data are shown as the mean \pm standard deviation.

Table 4 Postoperative blood routine and drainage indexes

\begin{tabular}{lcc}
\hline Variable* & Intubation group, $\mathrm{n}=75$ & Non-intubation group, $\mathrm{n}=117$ \\
\hline Blood routine, $24 \mathrm{~h}$ & & $\mathrm{P}$ \\
- WBC, $\times 10^{9}$ & $9.25 \pm 4.40$ & $7.67 \pm 2.15$ \\
- NEUT, \% & $7.06 \pm 4.02$ & $5.53 \pm 1.84$ \\
- LYMPH, \% & $1.45 \pm 0.45$ & $1.27 \pm 0.35$ \\
- HB, g/L & $126.93 \pm 18.53$ & $120.45 \pm 19.22$ \\
- PLT, $10^{9}$ & $229.65 \pm 69.95$ & $216.51 \pm 57.49$ \\
Oral intake chest drainage, hour & & 0.039 \\
$24 \mathrm{~h}$ & $198.43 \pm 127.24$ & 0.213 \\
$48 \mathrm{~h}$ & $114.04 \pm 109.48$ & 0.212 \\
\hline
\end{tabular}

*, postoperative indexes; the continuous data are shown as the mean \pm standard deviation; $\bullet$ WBC $\times 10^{9}, \bullet \mathrm{NEU} \%, \cdot \mathrm{LYMPH} \%, \bullet \mathrm{HB} \mathrm{g} / \mathrm{L}$, - PLT $\times 10^{9}$ indicate the difference of white blood cell, neutrophilic granulocyte percentage, lymphocytes percentage, hemoglobin, platelet count for the 2 groups after surgery.

surgery and was successful rehabilitation. I deem that these risk factors of non-intubation anesthesia VAST lung surgery can be overcome to establish clinical advantage.

Enhanced postoperative recovery (ERAS) was initially known in many other surgeries $(15,16)$. Non-intubated VATS lung surgery can optimize ERAS channel $(8,17)$. Applications of non-intubated VATS may be better integrated with ERAS strategies, our outcomes show that non-intubated VAST lung surgery combined with ERAS strategies is beneficial for the quickly recovery of postoperative patients. we found that compared with the intubation group, the non-intubation group was significantly earlier for the recovery of postoperative oral intake water (food) $[(3.52 \pm 0.72 v s .1 .12 \pm 0.38 \mathrm{~h})$ and (3.98 \pm 0.60 vs. $1.658 \pm 0.51 \mathrm{~h}), \mathrm{P}<0.001]$. Liu et al. believe that non-intubated anesthesia VAST segmentectomy, the patient resumed oral-intake 6 hours of postoperation (18), but our outcomes shown patients were able to resume oral-intake less than 2 hours after surgery, postoperative rehabilitation outcome is better. The out of bed activity time of postoperative patients is earlier $(11.54 \pm 2.44$ vs. $2.23 \pm 0.47$ h, $\mathrm{P}<0.001)$. In our view, pleural drainage was significantly 
reduced 24 and $48 \mathrm{~h}$ after recovery oral-intake, we consider that this is closely related to the early resumption of oralintake and ambulation, pleural drainage fluid is rapidly absorbed. The time of the (exhaust) defecation and total pleural drainage were shorter than the intubation group $(\mathrm{P}<0.05)$. However, hospital stays not exist significant difference, we think that the outcome caused by longer data collection period.

When the stress and inflammatory responses subside, patients quickly recover. It has been reported that during VATS lung surgery with non-intubated anesthesia, the postoperative inflammatory cell responses of patients were significantly lower than those of patients in the general anesthesia intubated group, which may be the result of inflammatory regulation (19). Another report (20) showed that the postoperative stress reaction and inflammatory cell reaction of non-intubated VATS lung surgery patients were relatively mild, which was related to the inhibition of afferent and efferent sympathetic pathways. We found that the $\downarrow$ WBC, $\downarrow$ NEUT, and $\downarrow$ LYMPH results of the nonintubated group after surgery were significantly lower than those of the intubated group.

Based on the comprehensive evaluation of this study, non-intubated anesthesia lung surgery accelerates the rapid postoperative recovery of patients. However, it should be noted that this retrospective study was conducted at a single-center and had a limited sample size, and thus some bias may exist. In addition, the mechanism underlying the rapid postoperative recovery of patients requires further study. We intend to examine this further in future studies at our center.

\section{Conclusions}

In summary, this study showed that non-intubated VATS lung surgery is a safe and beneficial option that encourages the rapid recovery of patients. Compared to intubation, the technique has a number of advantages in postoperative clinical rehabilitation. However, the correct administration and assessment for non-intubation anesthesia, multidisciplinary coordination and close cooperation during surgery are key factors related to its success. As this model promotes rapid patient recovery, it may represent an effective option for clinical surgeons in the future. As surgical teams gain experience, minimally invasive nonintubation anesthesia strategies can be extended to more challenging procedures.

\section{Acknowledgments}

Funding: The study was supported by Science and Technology Program of Kunming City (2020-1-H-003).

\section{Footnote}

Reporting Checklist: The authors have completed the STROBE reporting checklist. Available at https://dx.doi. org/10.21037/atm-21-6434

Data Sharing Statement: Available at https://dx.doi. org/10.21037/atm-21-6434

Conflicts of Interest: All authors have completed the ICMJE uniform disclosure form (Available at https://dx.doi. org/10.21037/atm-21-6434). The authors have no conflicts of interest to declare.

Ethical Statement: The authors are accountable for all aspects of the work in ensuring that questions related to the accuracy or integrity of any part of the work are appropriately investigated and resolved. Ethical approval for the study was granted by the Ethics Committee of The First People's Hospital of Yunnan Province (KHLL2021-KY091). In addition, written informed consent was obtained from all patients who participated in the study. All procedures performed in this study involving human participants were in accordance with the Declaration of Helsinki (as revised in 2013).

Open Access Statement: This is an Open Access article distributed in accordance with the Creative Commons Attribution-NonCommercial-NoDerivs 4.0 International License (CC BY-NC-ND 4.0), which permits the noncommercial replication and distribution of the article with the strict proviso that no changes or edits are made and the original work is properly cited (including links to both the formal publication through the relevant DOI and the license). See: https://creativecommons.org/licenses/by-nc-nd/4.0/.

\section{References}

1. Sunaga H, Blasberg JD, Heerdt PM. Anesthesia for nonintubated video-assisted thoracic surgery. Curr Opin Anaesthesiol 2017;30:1-6.

2. Tikka T, Hilmi OJ. Upper airway tract complications of endotracheal intubation. Br J Hosp Med (Lond) 
2019;80:441-7.

3. Gonzalez-Rivas D, Bonome C, Fieira E, et al. Nonintubated video-assisted thoracoscopic lung resections: the future of thoracic surgery? Eur J Cardiothorac Surg 2016;49:721-31.

4. Gelzinis TA, Sullivan EA. Non-Intubated General Anesthesia for Video-Assisted Thoracoscopic Surgery. J Cardiothorac Vasc Anesth 2017;31:407-8.

5. Gonzalez-Rivas D, Yang Y, Guido W, et al. Nonintubated (tubeless) uniportal video-assisted thoracoscopic lobectomy. Ann Cardiothorac Surg 2016;5:151-3.

6. Lirio F, Galvez C, Bolufer S, et al. Tubeless major pulmonary resections. J Thorac Dis 2018;10:S2664-70.

7. Liu Z, Yang R, Sun Y. Tubeless uniportal thoracoscopic wedge resection with modified air leak test and chest tube drainage. BMC Surg 2020;20:301.

8. Shen C, Che G. Tubeless minimally invasive treatment: taking a new step in enhanced recovery after surgery (ERAS). Thorac Cancer 2019;10:2067-70.

9. Semenkovich TR, Hudson JL, Subramanian M, et al. Enhanced Recovery After Surgery (ERAS) in Thoracic Surgery. Semin Thorac Cardiovasc Surg 2018;30:342-9.

10. Ljungqvist O. ERAS--enhanced recovery after surgery: moving evidence-based perioperative care to practice. JPEN J Parenter Enteral Nutr 2014;38:559-66.

11. Xu JY, Li YJ, Ning XG, et al. SV-VATS exhibits dual intraoperative and postoperative advantages. Ann Transl Med 2021;9:970.

12. He J, Liu J, Zhu C, et al. Expert consensus on tubeless video-assisted thoracoscopic surgery (Guangzhou). J Thorac Dis 2019;11:4101-8.

Cite this article as: Cai LS, Hou B, Jin H, Bo Y, Chen XL, Dai J, Yang T, Lan BS, Ye J, Peng H, Peng J. Clinical evaluation of the rapid recovery of patients who underwent video-assisted thoracoscopic lung surgery under non-intubated anesthesia. Ann Transl Med 2021;9(24):1783. doi: 10.21037/atm-21-6434
13. Zhang K, Chen HG, Wu WB, et al. Non-intubated videoassisted thoracoscopic surgery vs. intubated video-assisted thoracoscopic surgery forthoracic disease: a systematic review and meta-analysis of 1,684 cases. J Thorac Dis 2019;11:3556-68.

14. AlGhamdi ZM, Lynhiavu L, Moon YK, et al. Comparison of non-intubated versus intubated video-assisted thoracoscopic lobectomy for lung cancer. J Thorac Dis 2018;10:4236-43.

15. Batchelor TJP, Rasburn NJ, Abdelnour-Berchtold E, et al. Guidelines for enhanced recovery after lung surgery: recommendations of the Enhanced Recovery After Surgery (ERAS®) Society and the European Society of Thoracic Surgeons (ESTS). Eur J Cardiothorac Surg 2019;55:91-115.

16. Hubert J, Bourdages-Pageau E, Garneau CAP, et al. Enhanced recovery pathways in thoracic surgery: the Quebec experience. J Thorac Dis 2018;10:S583-90.

17. Umari M, Falini S, Segat M, et al. Anesthesia and fasttrack in video-assisted thoracic surgery (VATS): from evidence to practice. J Thorac Dis 2018;10:S542-54.

18. Liu HY, Chiang XH, Hung MH, et al. Nonintubated uniportal thoracoscopic segmentectomy for lung cancer. J Formos Med Assoc 2020;119:1396-404.

19. Peng J, An S, Wang HP, et al. Video-assisted thoracoscopic surgery lobectomy for lung cancer versus thoracotomy: a less decrease in sVEGFR2 level after surgery. J Thorac Dis 2016;8:323-8.

20. Vanni G, Tacconi F, Sellitri F, et al. Impact of awake videothoracoscopic surgery on postoperative lymphocyte responses. Ann Thorac Surg 2010;90:973-8. 